\title{
Invariant Subspaces and Exact Solutions to the Generalized Strongly Dispersive DGH Equation
}

\author{
Xuexia Li, Hanze Liu*, Lina Chang \\ School of Mathematical Sciences, Liaocheng University, Liaocheng, China \\ Email:877688025@qq.com, ^hnz_liu@aliyun.com,changlina2018@163.com
}

How to cite this paper: Li, X.X., Liu, H.Z. and Chang, L.N. (2020) Invariant Subspaces and Exact Solutions to the Generalized Strongly Dispersive DGH Equation. Journal of Applied Mathematics and Physics, 8, 1654-1663.

https://doi.org/10.4236/jamp.2020.88126

Received: July 19, 2020

Accepted: August 23, 2020

Published: August 26, 2020

Copyright $\odot 2020$ by author(s) and Scientific Research Publishing Inc. This work is licensed under the Creative Commons Attribution International License (CC BY 4.0).

http://creativecommons.org/licenses/by/4.0/

\begin{abstract}
In this paper, the invariant subspaces of the generalized strongly dispersive DGH equation are given, and the exact solutions of the strongly dispersive DGH equation are obtained. Firstly, transform nonlinear partial differential Equation (PDE) into ordinary differential Equation (ODE) systems by using the invariant subspace method. Secondly, combining with the dynamical system method, we use the invariant subspaces which have been obtained to construct the exact solutions of the equation. In the end, the figures of the exact solutions are given.
\end{abstract}

\section{Keywords}

Generalized Strongly Dispersive DGH Equation, Exact Solution, Invariant Subspace

\section{Introduction}

Nonlinear phenomena occur widely in various scientific fields, such as fluid mechanics, solid state physics, etc. As an important mathematical model to describe nonlinear phenomena, nonlinear partial differential equation has been widely concerned by many scholars in recent years, and the exact solutions of the PDE, including the soliton solution and wave solution, has always been a subject of interest to mathematical physicists. Recently, the methods of solving nonlinear partial differential equations mainly include Backlund-transformation method, non-locally symmetric method, Lie group method, and invariant subspace method, etc.

The invariant subspace method was first proposed by Galaktionov et al., and then extended by many scholars and widely applied. For example, precise solutions of Hunter-Saxton equation and compressible Euler equation were obtained. This method was derived from the Lie symmetry analysis, and it is re- 
lated to the conditional Lie-Bäcklund symmetry method [1] [2] and the differential constraint method; its key step is to transform a nonlinear PDE into ODE systems based on the invariant subspaces [3]. Then, we use the invariant subspaces we have obtained to construct the exact solutions of the nonlinearly PDE equations. So it is a dynamical system method by nature [3].

The outstanding feature of the invariant subspace method is its wide application range; it is also an algorithm that can construct more solutions and similar solutions for nonlinear partial differential equations.

However, there are still many problems in this method, such as how to construct more exact solutions according to different nonlinear equations. Furthermore, how to improve the efficiency of solving ordinary differential equations, such as with the help of Maple program, should be further studied.

In 1993, Camassa and Holm obtained a new class of completely integrable nonlinear shallow water wave equations Camassa-Holm $(\mathrm{CH})$ equation

$$
u_{t}+2 a u_{x}-u_{x x t}+3 u u_{x}=2 u_{x} u_{x x x}+u u_{x x x}
$$

Equation (1.1) has a double Hamilton structure and is completely integrable. When $a \neq 0$, it has smooth isolated wave solutions, when $a=0$, it has a peaked solitary wave solution the form of $u=c \mathrm{e}^{-|x-c t|}$ (where $c$ is the velocity).

Dullin, Gottwald and Holm derived the DGH equation from a class of shallow water wave equations of non-local asymptotic form [4]

$$
u_{t}+2 a u_{x}-u_{x x t}+3 u u_{x}+\gamma u_{x x x}=2 u_{x} u_{x x}+u u_{x x x}
$$

where $u_{x x}$ is the linear dispersive term, when $\gamma=0$, Equation (1.2) is the Camassa-Holm $(\mathrm{CH})$ equation, when $\left(u-u_{x x}\right)_{x x x}$ is the dispersive term [5], Equation (1.2) becomes the generalized strongly dispersive DGH equation

$$
u_{t}+2 c_{1} u_{x}-u_{x x t}+c_{2} u u_{x}+\gamma\left(u-u_{x x}\right)_{x x x}=2 u_{x} u_{x x}+u u_{x x x}
$$

where $u=u(x, t)$, is an unknown function, and $c_{1}, c_{2}, \gamma$ are arbitrary constants. Equation (1.3) describes the unidirectional propagation of surface waves in shallow water.

In recent years, many scholars have also done a lot of research on DGH equation. In 2007, Tian Lixin studied the global existence of the solution and Blow-up phenomenon and Hamilton structure of DGH equation. By using the qualitative analysis of planar autonomous system method, Guo Bolin and Liu Zhengrong obtained the spike isolated wave solutions of DGH equation. Compared with other methods, the invariant subspace method can obtain many new exact solutions of the generalized strongly dispersive DGH equation, including rational function solutions, which are different from symmetric reduction solutions and soliton solutions [6] [7] [8] [9]. The purpose of the current paper is to provide more exact solutions to the generalized strongly dispersive DGH Equation (1.3) by using the invariant subspace method. Firstly, we transform nonlinear partial differential Equation (PDE) into ordinary differential Equation (ODE) systems by using the invariant subspace method. Secondly, combining with the dynamical system method, we use the invariant subspaces which have obtained to con- 
struct the exact solutions of the generalized strongly dispersive DGH equation. Finally, the figures of the exact solutions are given.

This paper is organized as follows: In Section 2, the invariant subspace method is introduced briefly. In Section 3, all invariant subspaces and their basis functions are given. In Section 4, by using the results given in Section 3, the rational function solution, trigonometric function solution and exponential function solution of the Equation (1.3) are obtained. In Section 5, the conclusion and prospect are given.

In the following, we use $G$ to represent the m-order differential operator, and by using the invariant subspace method, giving the nonlinear evolution equation $F[u]$ determined by the differential operator and its corresponding invariant subspaces, and the basis functions in the invariant subspaces.

\section{Invariant Subspace Method}

Firstly, we consider a nonlinear evolution equation as follows:

$$
u_{t}=G[u] \equiv G\left[x, u, \frac{\partial u(x, t)}{\partial x}, \cdots, \frac{\partial^{n} u(x, t)}{\partial x^{n}}\right]
$$

where $u=u(x, t), G$ is a $m$-th order differential operator, if have $p$ functions $g_{1}(x), \cdots, g_{p}(x)$ are linearly independent, and the $n$-dimensional linear space is [10] [11]

$$
W_{p}=\mho\left\{g_{i}(x), g_{2}(x), \cdots, g_{p}(x)\right\}=\left\{\sum_{i=1}^{p} c_{i}(t) g_{i}(x), 1 \leq i \leq p\right\}
$$

If $G$ satisfies $G\left[W_{p}\right] \subseteq W_{p}$, that is, for any constant $c_{i}$, satisfy

$$
G\left(\sum_{i=1}^{p} c_{i} g_{i}(x)\right)=\sum_{i=1}^{p} G_{i}\left(c_{i}, c_{2}, \cdots, c_{p}\right) g_{i}(x)
$$

The invariant subspace $W_{p}$ is allowed by the operator $G$, so the nonlinear Equation (2.1) have the solution as follows

$$
u(x, t)=\sum_{i=1}^{p} c_{i}(t) g_{i}(x)
$$

where $c_{i}$ satisfy $n$-dimensional dynamical system [12] [13]

$$
c^{\prime}(t)=G\left(c_{1}(t), c_{2}(t), \cdots, c_{p}(t)\right), 1 \leq i \leq p
$$

Suppose the subspace $W_{p}$ composed of solutions to the following linear ODE

$$
L[y] \equiv y^{(p)}+a_{p-1}(x) y^{(p-1)}+\cdots+a_{1} y^{\prime}+a_{0} y=0
$$

then the invariant condition with respect to $G$ takes the form

$$
\left.L[G[u]]\right|_{L[u]=0} \equiv 0
$$

Theorem 1. If the subspace $W_{P}$ is invariant under a nonlinear differential operator $G$ of order $m$, then $p \leq 2 m+1$.

\section{Invariant Subspace of Equation (1.3)}

Firstly, Equation (1.3) can be written in the form of general evolution Equation 
$(2.1)$

$$
u_{t}-u_{x x t}=F[u]=2 u_{x} u_{x x}+u u_{x x x}-2 c_{1} u_{x}-c_{2} u u_{x}-\gamma\left(u-u_{x x}\right)_{x x x}
$$

the nonlinear operator $G$ on the right side of Equation (3.1) is

$$
G[u]=2 u_{x} u_{x x}+u u_{x x x}-2 c_{1} u_{x}-c_{2} u u_{x}
$$

then, by the invariant subspace theorem 1 , we have $p \leq 7$, in what follows, the invariant subspaces of Equation (1.3) are given.

\section{1. $p=2$}

In this case, the invariant condition of $W_{2}$ reads

$$
L_{2}\left[\left.G[u]\right|_{L_{2}[u]=0}=D^{2} G+a_{1} D G+\left.a_{0} G\right|_{L_{2}[u]=0}=0\right.
$$

where $L_{2}[u]=u^{\prime \prime}+a_{1} u^{\prime}+a_{0} u=0$, substituting Equation (3.2) into Equation (3.3), we have a polynomial in $u_{x}, u$

$$
\left(-6 a_{1}^{3}+10 a_{0} a_{1}+2 a_{1} c_{1}\right) u_{x}^{2}+\left(-11 a_{0} a_{1}^{2}+9 a_{0}^{2}+3 a_{0} c_{2}\right) u u_{x}-5 a_{0}^{2} a_{1} u^{2}=0 .
$$

take the coefficients of the above polynomial to be zero, we obtain the overdetermined equations for solving $a_{i}, c_{i}$

$$
\begin{aligned}
& u_{x}^{2}:-6 a_{1}^{3}+10 a_{0} a_{1}+2 a_{1} c_{1}=0 \\
& u u_{x}:-11 a_{0} a_{1}^{2}+9 a_{0}^{2}+3 a_{0} c_{2}=0 \\
& u^{2}: 5 a_{0}^{2} a_{1}=0
\end{aligned}
$$

By solving the above equations, we can get three sets of solutions as follows:

1) $a_{0}=0, a_{1}=a_{1}, c_{1}=c_{1}, c_{2}=3 a_{1}^{2}$;

2) $a_{0}=0, a_{1}=0, c_{1}=c_{1}, c_{2}=c_{2}$;

3) $a_{0}=a_{0}, a_{1}=0, c_{1}=c_{1}, c_{2}=-3 a_{0}$.

According to the above three solutions, the two-dimensional invariant subspaces allowed by the differential operator $G[u]$ of the Equation (3.2) are

$$
\begin{gathered}
G_{21}\left\{\begin{array}{l}
F[u]=u_{t}-u_{x x t}+\gamma\left(u-u_{x x}\right)_{x x x}=2 u_{x} u_{x x}+u u_{x x x}-2 c_{1} u_{x}-3 a_{1}^{2} u u_{x}, \\
L_{2}[y]=y^{\prime \prime}+a_{1} y=0, \\
W_{21}=\mho\left\{1, \exp \left(-a_{1} x\right)\right\} .
\end{array}\right. \\
G_{22}\left\{\begin{array}{l}
F[u]=u_{t}-u_{x x t}+\gamma\left(u-u_{x x}\right)_{x x x}=2 u_{x} u_{x x}+u u_{x x x}-2 c_{1} u_{x}-c_{2} u u_{x}, \\
L_{2}[y]=y^{\prime \prime}=0, \\
W_{22}=\mho\{1, x\} .
\end{array}\right. \\
G_{23}\left\{\begin{array}{l}
F[u]=u_{t}-u_{x x t}+\gamma\left(u-u_{x x}\right)_{x x x}=2 u_{x} u_{x x}+u u_{x x x}-2 c_{1} u_{x}+3 a_{0} u u_{x}, \\
L[y]=y^{\prime \prime}+a_{0} y=0, \\
W_{21}=\mho\left\{\cos \left(\sqrt{a_{0}} x\right), \sin \left(\sqrt{a_{0}} x\right)\right\}\left(a_{0}>0\right), \\
W_{23}=\mho\left\{\exp \left(-\sqrt{-a_{0}} x\right), \exp \left(\sqrt{-a_{0}} x\right)\right\}\left(a_{0}<0\right) .
\end{array}\right.
\end{gathered}
$$

\section{2. $p=3$}

In this case, the invariant condition of $W_{3}$ reads 


$$
\left.L[G[u]]\right|_{L_{3}[u]=0}=\left.\left(D^{3} G+a_{2} D^{2} G+a_{1} D G+a_{0} G\right)\right|_{L_{3}[u]=0}=0
$$

where $L_{3}[u]=u^{\prime \prime \prime}+a_{2} u^{\prime \prime}+a_{1} u^{\prime}+a_{0} u=0$, substituting the Equation (3.2) into the Equation (3.3), we have a polynomial in $u^{2}, u u_{x}, u_{x}^{2}, u u_{x x}, u_{x} u_{x x}, u_{x x}^{2}$, and take its coefficients to be zero, we obtain the overdetermined equations for solving $a_{i}, c_{i}$

$$
\begin{aligned}
& u^{2}: 7 a_{0}^{2}=0 \\
& u u_{x}: 18 a_{0} a_{2}=0 \\
& u_{x}^{2}:-a_{0} a_{2}^{2}+16 a_{0} a_{1}+4 a_{0} c_{2}=0 \\
& u u_{x x}:-a_{1} a_{2}^{2}+a_{0} a_{2}+9 a_{1}^{2}+3 a_{1} c_{2}=0 \\
& u_{x} u_{x x}:-a_{2}^{3}+21 a_{1} a_{2}+a_{2} c_{2}-14 a_{0}=0 \\
& u_{x x}^{2}: 11 a_{2}^{2}-9 a_{1}-3 c_{2}=0
\end{aligned}
$$

By solving the above equations, we get a unique set of solutions as follows

$$
\left\{a_{0}=0, a_{1}=a_{1}, a_{2}=0, c_{1}=c_{1}, c_{2}=-3 a_{1}\right\}
$$

Thus, we obtain the three-dimensional invariant subspace is

$$
G_{3}\left\{\begin{array}{l}
F[u]=u_{t}-u_{x x t}+\gamma\left(u-u_{x x}\right)_{x x x}=2 u_{x} u_{x x}+u u_{x x x}-2 c_{1} u_{x}+3 a_{1} u u_{x}, \\
L[y]=y^{\prime \prime \prime}+a_{1} y^{\prime}=0, \\
W_{31}=\mho\left\{1, \cos \left(\sqrt{a_{1} x}\right), \sin \left(\sqrt{a_{1}} x\right)\right\}\left(a_{1}>0\right), \\
W_{32}=\mho\left\{1, \exp \left(-\sqrt{-a_{1}} x\right), \exp \left(\sqrt{-a_{1} x}\right)\right\}\left(a_{1}<0\right) .
\end{array}\right.
$$

From the invariant subspace obtained when $n=2$ and $n=3$, we can see that the invariant subspace method is only related to the nonlinear terms of the equation. According to the similar calculation steps above, we can get the invariant subspaces when $p=4,5,6$. But, when $p=7$, there is no solution.

\section{3. $p=4$}

The four-dimensional invariant subspace allowed by the differential operator $G[u]$ of the Equation (3.2) is

$$
G_{4}=\left\{\begin{array}{l}
F[u]=u_{t}-u_{x x t}+\gamma\left(u-u_{x x}\right)_{x x x}=2 u_{x} u_{x x}+u u_{x x x}-2 c_{1} u_{x}, \\
L[y]=y^{4}=0 \\
W_{4}=\mho\left\{1, x, x^{2}, x^{3}\right\}
\end{array}\right.
$$

\section{4. $p=5$}

The five-dimensional invariant subspace allowed by the differential operator $G[u]$ of the Equation (3.2) is

$$
G_{5}=\left\{\begin{array}{l}
F[u]=u_{t}-u_{x x t}+\gamma\left(u-u_{x x}\right)_{x x x}=2 u_{x} u_{x x}+u u_{x x x}-2 c_{1} u_{x}, \\
L[y]=y^{5}=0, \\
W_{5}=\mho\left\{1, x, x^{2}, x^{3}, x^{4}\right\} .
\end{array}\right.
$$

\section{5. $p=6$}

The six-dimensional invariant subspace allowed by the differential operator 
$G[u]$ of the Equation (3.2) is

$$
G_{6}=\left\{\begin{array}{l}
F[u]=u_{t}-u_{x x t}+\gamma\left(u-u_{x x}\right)_{x x x}=2 u_{x} u_{x x}+u u_{x x x}-2 c_{1} u_{x}+3 a_{1} u u_{x}, \\
L[y]=y^{6}=0, \\
W_{6}=\mho\left\{1, x, x^{2}, x^{3}, x^{4}, x^{5}\right\} .
\end{array}\right.
$$

\section{Exact Solutions and Figures of Equation (1.3)}

In this section, combining the invariant subspace method with the dynamical system method, by using the results obtained in Section 3, construing the exact solution of the Equation (1.3) in invariant subspaces. We can divide the solutions of generalized strongly dispersive DGH equation into three types: polynomial function solutions, exponential function solutions and trigonometric function solutions. Then, the figures of the exact solutions are given.

\subsection{Polynomial Solution of the Equation (1.3)}

When $n=2$, for

$$
F[u]=u_{t}-u_{x x t}+\gamma\left(u-u_{x x}\right)_{x x x}=2 u_{x} u_{x x}+u u_{x x x}-2 c_{1} u_{x}-c_{2} u u_{x}
$$

where $c_{1}, c_{2}, \gamma$ are arbitrary constants, from $G_{22}$ in Section 3, we get $W_{22}=\mho\{1, x\}$.

So, we can suppose that Equation (4.1) has the solution as follows:

$$
u_{1}=\phi_{0}(t)+\phi_{1}(t) x
$$

Substituting (4.2) into Equation (4.1), we get the equation for $x^{n}(n=0,1)$, and take its coefficients to be zero, we get

$$
\left\{\begin{array}{l}
\phi_{2}^{\prime}(t)-\phi_{2}^{2}(t) c_{2}=0, \\
\phi_{1}^{\prime}(t)-\phi_{1}(t) \phi_{2}(t) c_{2}-2 c_{1} \phi_{2}(t)=0 .
\end{array}\right.
$$

By solving Equation (4.3), we get

$$
\phi_{1}(t)=\frac{2 c_{1} t \pm C_{2}}{-C_{2} t \pm C_{1}}, \phi_{2}(t)=\frac{1}{-c_{2} t \pm C_{1}}
$$

The Polynomial solution of Equation (4.1) are obtained

$$
u_{1}=\frac{2 c_{1} t+C_{2}}{-C_{2} t+C_{1}}+\frac{1}{-c_{2} t+C_{1}} x
$$

See Figure 1(a) and Figure 1(b).

Figure 1(a) is the rational solution of the Equation (4.1) when $c_{1}=-2, c_{2}=-1$, $C_{1}=C_{2}=1$, and $x=-10 \sim 10, t=-10 \sim 10$.

Figure 1(b) is the rational solution of the Equation (4.1) when $C_{1}=c_{2}=C_{1}=C_{2}=1$, and $x=-10 \sim 10, t=-10 \sim 10$.

\subsection{Trigonometric Solution and Exponential Solution of Equation} (1.3)

When $n=3$, for 


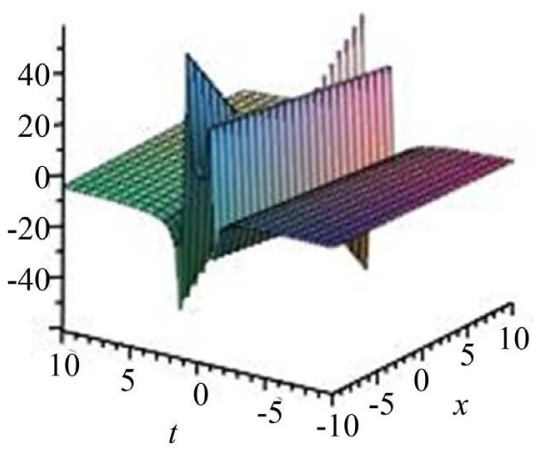

(a)

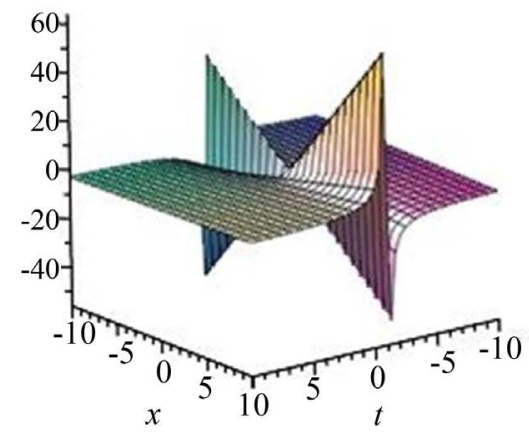

(b)

Figure 1. Polynomial solution of Equation (4.1).

$$
F[u]=u_{t}-u_{x x t}+\gamma\left(u-u_{x x}\right)_{x x x}=2 u_{x} u_{x x}+u u_{x x x}-2 c_{1} u_{x}+3 a_{1} u u_{x}
$$

where $c_{1}, c_{2}, \gamma$ are arbitrary constants. Suppose that Equation (4.4) has exact solutions in the form of trigonometric and exponential functions:

$$
\begin{gathered}
u_{2,1}=\phi_{0}(t)+\phi_{1}(t) \cos \left(\sqrt{a_{1}} x\right)+\phi_{2}(t) \sin \left(\sqrt{a_{1}} x\right), a_{1}>0, \\
u_{2,2}=\phi_{0}(t)+\phi_{1}(t) \exp \left(\sqrt{-a_{1}} x\right)+\phi_{2}(t) \exp \left(-\sqrt{-a_{1}} x\right), a_{1}<0 .
\end{gathered}
$$

substituting (4.5) into Equation (4.4), we get the equation for $\cos \left(\sqrt{a_{1}} x\right), \sin \left(\sqrt{a_{1}} x\right)$, and take its coefficients to be zero, we get

$$
\left\{\begin{array}{l}
\left(1+a_{1}\right) \phi_{2}^{\prime}(t)+\gamma a_{1}^{\frac{5}{2}} \phi_{1}(t)-2 a_{1}^{\frac{3}{2}} \phi_{0}(t) \phi_{1}(t)-\gamma a_{1}^{\frac{3}{2}} \phi_{1}(t)-2 \sqrt{a_{1}} c_{1} \phi_{1}(t)=0 \\
\left(1+a_{1}\right) \phi_{1}^{\prime}(t)-\gamma a_{1}^{\frac{5}{2}} \phi_{2}(t)+2 a_{1}^{\frac{3}{2}} \phi_{0}(t) \phi_{2}(t)-\gamma a_{1}^{\frac{3}{2}} \phi_{2}(t)-2 \sqrt{a_{1}} c_{1} \phi_{2}(t)=0 \\
\phi_{0}^{\prime}(t)=0
\end{array}\right.
$$

By solving Equation (4.3), we get

$$
\phi_{0}(t)=C_{1}, \phi_{1}(t)=C_{2} \sin (A t)+C_{3} \cos (A t), \phi_{2}(t)=C_{2} \cos (A t)-C_{3} \sin (A t)
$$

So, the solution in form of the trigonometric function of Equation (4.4) is

$$
\begin{aligned}
u_{2,1}= & C_{1}+\left[C_{2} \sin (A t)+C_{3} \cos (A t)\right] \cos \left(\sqrt{a_{1}} x\right) \\
& +\left[C_{2} \cos (A t)-C_{3} \sin (A t)\right] \sin \left(\sqrt{a_{1}} x\right) .
\end{aligned}
$$

where $C_{1}, C_{2}, C_{3}$ are arbitrary constants, and $A=\frac{\left(a_{1}^{2} \gamma-2 C_{1} a_{1}+a_{1} \gamma+c_{1}\right) \sqrt{a_{1}} t}{a_{1}+1}$.

Substituting (4.6) into Equation (4.4), we get the equation for $\exp \left(\sqrt{-a_{1}} x\right), \exp \left(-\sqrt{-a_{1}} x\right)$, and take its coefficients to be zero, we get

$$
\left\{\begin{array}{l}
\gamma \sqrt{-a_{1}} \phi_{1} a_{1}^{2}-2 \phi_{0} \sqrt{-a_{1}} \phi_{1} a_{1}+\gamma \sqrt{-a_{1}} \phi_{1} a_{1}+2 \sqrt{-a_{1}} c_{1} \phi_{1}+\left(a_{1}+1\right) \phi_{1}^{\prime}=0 \\
-\gamma \sqrt{-a_{1}} \phi_{2} a_{1}^{2}+2 \phi_{0} \sqrt{-a_{1}} \phi_{1} a_{1}-\gamma \sqrt{-a_{1}} \phi_{2} a_{1}-2 \sqrt{-a_{1}} c_{1} \phi_{2}+\left(a_{1}+1\right) \phi_{2}^{\prime}=0 \\
\phi_{0}^{\prime}=0
\end{array}\right.
$$

By solving Equation (4.8), we get

$$
\phi_{0}(t)=C_{1}, \phi_{1}(t)=\frac{C_{2} \exp (B t)}{\exp (D t)}, \phi_{2}(t)=\frac{C_{3} \exp (D t)}{\exp (B t)}
$$




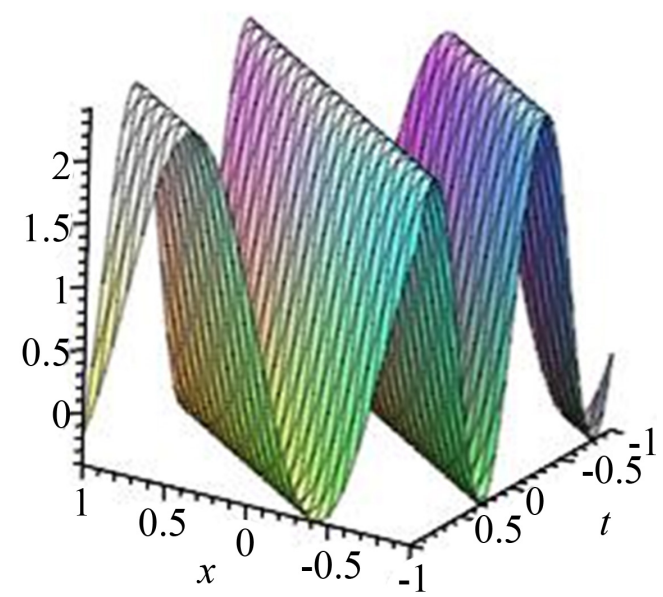

Figure 2. Trigonometric solution of Equation (4.4).

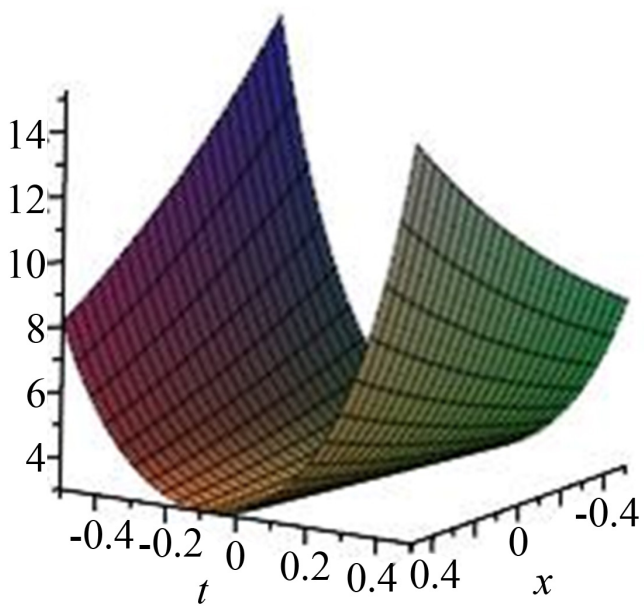

Figure 3. Exponential solution of Equation (4.4).

So, the trigonometric solution of Equation (4.4) is

$$
u_{2,2}=C_{1}+\frac{C_{2} \exp (B t)}{\exp (D t)} \exp \left(\sqrt{-a_{1}} x\right)+\frac{C_{3} \exp (D t)}{\exp (B t)} \exp \left(-\sqrt{-a_{1}} x\right) .
$$

where $C_{1}, C_{2}, C_{3}$ are arbitrary constants, and $B=\frac{2 C_{1} a_{1} \sqrt{-a_{1}}}{a_{1}+1}$,

$$
D=\frac{\left(a_{1}^{2} \gamma+a_{1} \gamma+2 c_{1}\right) \sqrt{-a_{1}}}{a_{1}+1} .
$$

See Figure 2 and Figure 3.

\section{Conclusion}

Firstly, by using the invariant subspace method, the invariant subspaces of the generalized strongly dispersive DGH equation are obtained. Secondly, we selected the partial invariant subspaces, and we get an exact solution other than the soliton of the equation, such as the solutions in form of polynomial function, trigonometric function and exponential function of the generalized strongly dis- 
persive DGH equation. Meanwhile, the invariant subspace method derived from symmetry analysis, this method also can be used to solve the nonlinear PDEs. And symmetry analysis is an invariance analysis to some extent. The above theory provides a direction for future research. Moreover, the results in the present paper are verified by the maple procedure.

\section{Acknowledgements}

The authors would like to thank the Editor and Reviewers for their valuable comments.

\section{Conflicts of Interest}

The authors declare no conflicts of interest regarding the publication of this paper.

\section{References}

[1] Ji, L.N. and Qu, C.Z. (2013) Conditional Lie-Bäcklund Symmetries and Invariant Subspaces to Nonlinear Diffusion Equations with Convection and Source. Studies in Applied Mathematics, 131, 266-301. https://doi.org/10.1111/sapm.12010

[2] Qu, C.Z. and Ji, L.N. (2013) Invariant Subspaces and Conditional Lie-Bäcklund Symmetries of Inhomogeneous Non-Linear Diffusion Equations. Studies in Applied Mathematics, 56, 2187-2203. https://doi.org/10.1007/s11425-013-4714-X

[3] Chang, L.N., Liu, H.Z. and Xin, X.P. (2020) Invariant Subspace Classification and Exact Explicit Solutions to a Class of Nonlinear Wave Equation. Qualitative Theory of Dynamical Systems, 19, Article No. 65. https://doi.org/10.1007/s12346-020-00400-6

[4] Camaasa, R. and Holm, D. (1993) An Integrable Shallow Water Equation with Peaked Solitons. Physical Review Letters, 13, 166l-1665.

[5] Galaktionov, V.A. (1995) Invariant Subspaces and New Explicit Solutions to Evolution Equations with Quadratic Nonlinearities. Proceedings of the Royal Society of Edinburgh Section A: Mathematics, 125, 225-246. https://doi.org/10.1017/S0308210500028018

[6] Galaktionov, A. and Svirshchevskii, S.R. (2007) Exact Solutions and Invariant Subspaces of Nonlinear Partial Differential Equations in Mechanics and Physics. Chapman and Hall, London. https://doi.org/10.1201/9781420011623

[7] Qu, C.Z. and Zhu, C.R. (2009) Classification of Coupled Systems with Two-Component Nonlinear Diffusion Equations by the Invariant Subspace Method. Journal of Physics A: Mathematical and Theoretical, 42, 1-27. https://doi.org/10.1088/1751-8113/42/47/475201

[8] Liu, H.Z. and Li, J.B. (2014) Symmetry Reductions, Dynamical Behavior and Exact Explicit Solutions to the Gordon Types of Equations. Journal of Computational and Applied Mathematics, 257, 144-156. https://doi.org/10.1016/j.cam.2013.08.022

[9] Shen, S.F. and Qu, C.Z. (2012) Maximal Dimension of Invariant Subspaces to Systems of Nonlinear Evolution Equations. Chinese Annals of Mathematics, Series B, 33, 161-178. https://doi.org/10.1007/s11401-012-0705-4

[10] Ma, W.X. (2012) A Refined Invariant Subspace Method and Applications to Evolution Equations. Science China Mathematics, 55, 1769-1778. 
https://doi.org/10.1007/s11425-012-4408-9

[11] Guo, Z.H., Liu, X.C., Liu, X.X. and Qu, C.Z. (2019) Stability of Peakons for the Generalized Modified Camassa-Holm Equation. Journal of Differential Equations, 266, 7749-7779. https://doi.org/10.1016/j.jde.2018.12.014

[12] Zhu, C.R. and Qu, C.Z. (2009) Classification and Reduction of Generalized Thin Film Equations. Communications in Theoretical Physics, 52, 403-410. https://doi.org/10.1088/0253-6102/52/3/05

[13] Liu, H.Z. (2018) Invariant Subspace Classification and Exact Solutions to the Generalized Nonlinear D-C Equation. Applied Mathematics Letters, 83, 164-168. https://doi.org/10.1016/j.aml.2018.03.032 